\title{
Treewidth Lower Bounds with Brambles
}

\author{
Hans L. Bodlaender • Alexander Grigoriev • \\ Arie M.C.A. Koster
}

Received: 22 December 2005 / Accepted: 6 November 2006 / Published online: 6 October 2007

(C) Springer Science+Business Media, LLC 2007

\begin{abstract}
In this paper we present a new technique for computing lower bounds for graph treewidth. Our technique is based on the fact that the treewidth of a graph $G$ is the maximum order of a bramble of $G$ minus one. We give two algorithms: one for general graphs, and one for planar graphs. The algorithm for planar graphs is shown to give a lower bound for both the treewidth and branchwidth that is at most a constant factor away from the optimum. For both algorithms, we report on extensive computational experiments that show that the algorithms often give excellent lower bounds, in particular when applied to (close to) planar graphs.
\end{abstract}

Keywords Treewidth · Lower bound · Bramble · Planar graph · Grid minor · Approximation algorithm

Communicated by Prof. Dr. Susanne Albers.

This work was partially supported by the Netherlands Organisation for Scientific Research NWO (project Treewidth and Combinatorial Optimisation) and partially by the DFG research group “Algorithms, Structure, Randomness" (Grant number GR 883/9-3, GR 883/9-4).

H.L. Bodlaender

Department of Information and Computing Sciences, Utrecht University, P.O. Box 80.089, 3508 TB Utrecht, The Netherlands

e-mail: hansb@cs.uu.nl

\section{A. Grigoriev $(\varangle)$}

Department of Quantitative Economics, University of Maastricht, P.O. Box 616, 6200 MD Maastricht, The Netherlands

e-mail: a.grigoriev@ke.unimaas.nl

A.M.C.A. Koster

Zuse Institute Berlin (ZIB), Takustraße 7, 14195 Berlin-Dahlem, Germany

e-mail: koster@zib.de 


\section{Introduction}

Motivation In many applications of the notion of treewidth, it is desirable that we can compute tree decompositions of small width of given graphs. Unfortunately, finding a tree decomposition of minimum width and determining the exact treewidth are NP-hard; see [3]. Much research has been done in recent years on the problem to determine the treewidth of the graph: this includes a faster exponential time algorithm [20], a theoretically optimal but due to the large constant factor hidden in the $O$-notation impractical linear time algorithm for the fixed parameter case [5], a polynomial time algorithm for graphs with polynomially many minimal separators [13], a branch and bound algorithm [22], preprocessing methods [9, 11], upper bound heuristics [2, 15, 27], and lower bound heuristics [10, 14, 28, 29, 31]. An overview with many references can be found in [7].

In this paper, we focus on lower bound methods for treewidth. Lower bound algorithms are interesting and useful for a number of different reasons. When running a branch and bound algorithm to compute the treewidth of a graph, see e.g. [22], a good lower bound helps to quickly cut off branches. Lower bounds inform us on the quality of upper bounds. Also, a high lower bound can tell that we should not aim for a solution of a problem on a certain graph instance with treewidth techniques: Suppose we decide we want to solve a certain problem on a given graph with a dynamic programming algorithm on a tree decomposition. If we have a large lower bound for the treewidth of that graph, we know in advance that this dynamic programming algorithm will use much time and memory, and hence we should direct our attention to trying different methods. Finally, better lower bounds for treewidth sometimes help to obtain further reductions in the size of graphs obtained by preprocessing [11].

In recent years, several treewidth lower bound methods have been found and evaluated. A trivial lower bound for the treewidth is the minimum degree of a vertex. Better lower bounds are obtained by looking at the minimum degrees of induced subgraphs or of graphs obtained by contractions, see [10]. Often slightly better than the minimum degree is a lower bound by Ramachandramurthi [31], which is (for noncomplete graphs) the minimum over all pairs of non-adjacent vertices $v, w$, of the maximum degree of $v$ and $w$. Another lower bound, found by Lucena [29] and analyzed in [8], is based on maximum cardinality search. Combining these bounds with contractions gives often considerable improvements [10, 28]. Further improvements can be obtained by using these techniques in combination with a method introduced by Clautiaux et al. [14], based upon adding edges between vertices that have many common neighbors or disjoint paths between them [12].

The experiments carried out in $[10,28]$ show that for several graphs, the existing lower bound methods give good bounds that are often close and in several cases equal to the actual treewidth. However, there are classes of graphs where each of these methods yields a rather small lower bound that is far away from the real treewidth. For instance, this holds for planar graphs or graphs that are in a certain sense close to being planar, e.g., graphs obtained by taking the union of a small number of TSPtours on a point set in the plane [16]. The reason that the above mentioned techniques appear to fail for these graphs mostly is due to the fact that all these methods in a certain sense are (minimum) degree-based, and (close to) planar graphs always have vertices of small degree, cf. [41]. 
For planar graphs, Seymour and Thomas [38] presented an $O\left(n^{2}\right)$ time algorithm computing a minimum branchwidth. Branchwidth of a graph is a lower bound for the treewidth, and moreover, the branchwidth of a graph approximates the treewidth within a factor of 3/2, see Robertson and Seymour [34]. Unfortunately, the algorithm of Seymour and Thomas requires a substantial, although polynomial, amount of memory, so it runs out of memory even for the medium size graphs (from 2000 nodes), see [24, 25]. In the later two papers, Hicks proposed another algorithm that at cost of slowing down the algorithm of Seymour and Thomas makes it "memory friendly". The algorithm of Hicks runs in time $O\left(n^{3}\right)$ and can deal with graphs up to 15000 nodes.

Since the known algorithms for a treewidth lower bound are either slow, or not memory friendly, or provide a bad quality bound, we were searching for a new method using a different principle that works well for graphs that are planar or close to planar. In this paper, we present such a different method, based on the notion of bramble (for the first time, brambles appeared in [37] with the name screens).

Notations and Preliminaries Throughout this paper, $G=(V, E)$ denotes an undirected simple graph. We use many of the standard graph theoretic notions in the usual way, like path, connected subgraph, etc. For a subset $S \subseteq V$ of the vertices, we denote with $N(S)$ the neighbors of $S$ that are outside $S$, i.e., $N(S)=\{w \in V \backslash S:\{v, w\} \in$ $E, v \in S\}$. With $G[S]$ we denote the subgraph of $G$ induced by $S \subseteq V$. Instead of $G[V \backslash S]$ we also write $G \backslash S$.

The vertex connectivity of non-adjacent vertices $v, w \in V$ in $G$ is defined as the minimum size of a vertex set $S \subset V$ such that $v$ and $w$ are in different components of $G \backslash S$. By Menger's Theorem [30], the number of vertex disjoint paths between $v$ and $w$ equals the vertex connectivity. The vertex connectivity of a pair $v, w$ and the vertex disjoint paths can be computed by maximum flow techniques, see e.g. [1].

The notions of tree decomposition and treewidth were first defined by Robertson and Seymour [33]. Several other, equivalent notions, have been proposed by many different authors, see e.g., [6].

Definition 1.1 A tree decomposition of a graph $G=(V, E)$ is a pair $\left(\left\{X_{i} \mid i \in I\right\}\right.$, $T=(I, F))$, with $\left\{X_{i} \mid i \in I\right\}$ a family of subsets of $V$ and $T$ a tree, such that

- $\bigcup_{i \in I} X_{i}=V$,

- For all $\{v, w\} \in E$, there is an $i \in I$ with $v, w \in X_{i}$, and

- For all $v \in V$, the set $I_{v}=\left\{i \in I \mid v \in X_{i}\right\}$ forms a connected subtree of $T$.

The width of a tree decomposition $\left(\left\{X_{i} \mid i \in I\right\}, T=(I, F)\right)$ is $\max _{i \in I}\left|X_{i}\right|-1$. The treewidth of a graph $G, t w(G)$, is the minimum width among all tree decompositions of $G$.

A graph $H$ is a minor of $G$, if $H$ can be obtained from $G$ by a series of zero or more vertex deletions, edge deletions, and edge contractions. It is well known that if $H$ is a minor of $G$, then the treewidth of $H$ is at most the treewidth of $G$.

A notion closely related to treewidth is the branchwidth of a graph, also introduced by Robertson and Seymour [34, 35]. 
Definition 1.2 A branch decomposition of a graph $G=(V, E)$ is a pair $(T=$ $(I, F), \sigma)$, with $T$ a ternary tree (a tree where every non-leaf node has degree 3 ) with $|E|$ leaves, and $\sigma$ a bijection from $E$ to the set of leaves of $T$.

The order of an edge $f \in F$ is the number of vertices $v \in V$, for which there exist adjacent edges $\{v, w\},\{v, x\} \in E$, such that the path in $T$ from $\sigma(v, w)$ to $\sigma(v, x)$ uses $f$.

The width of branch decomposition $(T=(I, F), \sigma)$, is the maximum order over all edges $f \in F$. The branchwidth of a graph $G, b w(G)$, is the minimum width over all branch decompositions of $G$.

Robertson and Seymour [34] proved that $\max \{b w(G), 2\} \leq t w(G)+1 \leq$ $\max \{\lfloor 3 b w(G) / 2\rfloor, 2\}$. One easily can convert a branch decomposition into a tree decomposition such that the respective widths fulfill these inequalities.

We next give the definitions of bramble and related notions, following the terminology of Reed [32].

Definition 1.3 Let $G=(V, E)$ be a graph. Two subsets $W_{1}, W_{2} \subseteq V$ are said to touch if they have a vertex in common or $E$ contains an edge between them $\left(W_{1} \cap W_{2} \neq \varnothing\right.$ or there is an edge $\left\{w_{1}, w_{2}\right\} \in E$ with $w_{1} \in W_{1}, w_{2} \in W_{2}$ ). A set $\mathcal{B}$ of mutually touching connected vertex sets is called a bramble. A subset of $V$ is said to cover $\mathcal{B}$ if it is a hitting set for $\mathcal{B}$ (i.e., a set which intersects every element of $\mathcal{B}$.) The order of a bramble $\mathcal{B}$ is the minimum size of a hitting set for $\mathcal{B}$. The bramble number of $G$ is the maximum order of all brambles of $G$.

The relationship between the bramble number and the treewidth was obtained by Seymour and Thomas [37]:

Theorem 1.4 (Seymour and Thomas [37]) Let $k$ be a non-negative integer. A graph has treewidth $k$ if and only if it has bramble number $k+1$.

For a short proof of this theorem we refer to Bellenbaum and Diestel [4].

Corollary 1.5 Given a bramble $\mathcal{B}$ of order $k$ in a graph $G, t w(G) \geq k-1$.

So, finding a high order bramble immediately implies getting a good lower bound for the treewidth. Unfortunately, determining the order of a bramble, is also NP-hard; it follows directly from the NP-hardness of the minimum hitting set problem [21] by taking a complete graph $G$ and the collection of subsets as the bramble.

A class of graphs for which the bramble of maximum order can be constructed easily is the class of grid graphs. It is folklore that an $x$ by $x$ grid has treewidth $x$; see e.g., Fig. 1 for a bramble of order $x+1$. We have one set that contains all vertices on the bottom row, one set that contains all vertices on the last column except the last one, and $(x-1)^{2}$ crosses, each consisting of the first $x-1$ vertices of one of the first $x-1$ rows and the first $x-1$ vertices of one of the first $x-1$ columns. A set that covers the bramble must contain at least $x-1$ vertices to cover the crosses, and one vertex in each of the other two sets. 
Fig. 1 Illustrating a bramble of a grid

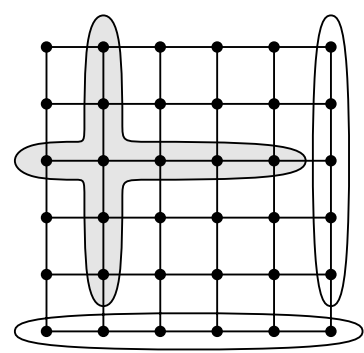

In this paper, we therefore restrict our search to brambles for which the order can be computed easily, due to the construction of the bramble. In Sect. 2 we construct brambles for general graphs; and in Sect. 3 a more sophisticated, though fast, algorithm for planar graphs is presented. The latter algorithm is a constant approximation algorithm for both treewidth and branchwidth of planar graphs. In Sect. 4, we report on computational results, showing the effectiveness of the brambles as lower bound for treewidth in those cases that the graph is (close to) planar. We finish the paper with some concluding remarks.

\section{Brambles in General Graphs}

In this section, we present Algorithm $\mathcal{A}_{1}$. Algorithm $\mathcal{A}_{1}$ gets as input an arbitrary undirected graph $G$, finds several brambles of $G$ or of minors of $G$, and outputs the best treewidth lower bound found this way.

First, in an initialization step, the set of vertices is split in a number of level sets, $V_{0}, \ldots, V_{R}$ (with $R$ defined within the initialization). For simplicity, the level sets are called by their levels. In the basic step, a number of brambles is constructed, and their order is computed. The maximum order encountered is recorded and returned at the end.

Before we can give a detailed description of the algorithm, we have to introduce some additional notation. Given a subset $S \subseteq V$ of the vertices with $G[S]$ not connected, we call a set $T$ a connectivity closure of $S$ in $G$ if $G[S \cup T]$ is connected. If $G$ is not connected, there does not exist a connectivity closure of $S$ in $G$.

A, not necessarily minimal, connectivity closure is provided by the following procedure: Let $T:=\varnothing$ in the beginning. Construct a bidirectional graph $D=(V, A)$ with two arcs for every edge in $E$. For $(v, w) \in A$, define a length of 0 if $v, w \in S \cup T$ and 1 otherwise. Now, compute the shortest path from an arbitrary vertex $v \in S \cup T$ to all other vertices in $S$. The distance between two vertices is either zero or at least two. If the length of a path between $v$ and $w$ is at least two, $v$ and $w$ are in two different components of $G[S \cup T]$, and the distance minus one is the minimum number of vertices required to connect both components. Now, let $w$ be a vertex for which the distance is minimum among those with distance at least two. We add the vertices on the $(v, w)$-path to $T$ and restart the procedure from the construction of $D$, until no distance between vertices of $S \cup T$ exceeds zero.

Now, we are ready to define the algorithm. 


\section{Algorithm $\mathcal{A}_{1}$}

Initialization.

1. Take an arbitrary vertex $r$ in $V$. Define $V_{0}:=\{r\}$. Set $k:=1$.

2. Let $V_{k}^{\prime}=N\left(V_{k-1}\right) \backslash\left\{\bigcup_{i=0}^{k-1} V_{i}\right\}$ be the vertices adjacent from $V_{k-1}$ that are not part of the existing subsets. If $G\left[V_{k}^{\prime}\right]$ is connected, then $V_{k}:=V_{k}^{\prime}$. Otherwise, we search for a connectivity closure $V_{k}^{\prime \prime}$ of $V_{k}^{\prime}$ in $G \backslash\left\{\bigcup_{i=0}^{k-1} V_{i}\right\}$. If such a closure exists, let $V_{k}:=V_{k}^{\prime} \cup V_{k}^{\prime \prime}$. If there is no connectivity closure for the set $V_{k}^{\prime}$, then redefine $V_{k-1}:=V \backslash\left\{\bigcup_{i=0}^{k-2} V_{i}\right\}$ and go to Step 3 of the initialization. In all other cases, set $k:=k+1$ and return to the beginning of Step 2 .

3. Set $R:=k$. Add to the graph a dummy vertex $q$; connect all vertices from the level $R-1$ to $q$; and define $V_{R}:=\{q\}$.

4. Initialize an integer variable bestlow to -1 .

Basic Step. For all $1 \leq i \leq j \leq R-1$ do the following.

1. Let $G_{i, j}$ be the graph resulting from $G$ after contraction of the first $i$ levels $V_{0}, \ldots, V_{i-1}$ into vertex $s_{i}$ and contraction of the last $R-j$ levels $V_{j+1}, \ldots, V_{R}$ into vertex $t_{j}$.

2. Let $c_{i j}$ be the vertex connectivity of the pair $\left(s_{i}, t_{j}\right)$ in $G_{i, j}$. Moreover, let $P_{\ell}$, $\ell=1, \ldots, c_{i j}$, denote the internal vertices of $c_{i j}$ vertex disjoint paths between $s_{i}$ and $t_{j}$.

3. Define $\mathcal{B}_{i, j}$ as follows. Let $\left\{s_{i}\right\}$ be an element of $\mathcal{B}_{i, j}$. For all $i \leq k \leq j$ and $1 \leq \ell \leq c_{i, j}$ let the set $V_{k} \cup P_{\ell}$ be an element of $\mathcal{B}_{i, j}$.

4. Set bestlow to the maximum of bestlow and $\min \left\{c_{i, j}, j-i+1\right\}$.

Output. Output $L B_{1}=$ bestlow. STOP.

Notice, that the procedure described in Step 2 of the initialization is a classical Breadth-First-Search (BFS) extended with taking connectivity closures. Moreover, note that for $1 \leq i \leq j \leq R-1$, the vertices $s_{i}$ and $t_{j}$ are not adjacent in $G_{i, j}$ by definition of the level sets, and thus the vertex connectivity is defined for these vertex pairs.

Theorem 2.1 The set $\mathcal{B}_{i, j}, 1 \leq i \leq j \leq R-1$, is a bramble of $G_{i, j} \backslash\left\{t_{j}\right\}$. The order of this bramble equals $\min \left\{c_{i, j}+1, j-i+2\right\}$.

Proof Take any pair $i, j$ such that $1 \leq i \leq j \leq R-1$ and consider the corresponding graph $G_{i, j}$. Let us check whether all conditions in the definition of brambles are satisfied for the set $\mathcal{B}_{i, j}$.

First of all, let us verify that each element of $\mathcal{B}_{i, j}$ induces a connected set. From initialization we know that each level is a connected set. The paths $P_{\ell}$ constructed in the basic step of $\mathcal{A}_{1}$ are clearly connected and each of those paths crosses all levels in $G_{i, j}$. Hence a union of any level and any path forms a connected set.

Secondly, all elements of $\mathcal{B}_{i, j}$ are mutually touching: all unions of level and path are touching $\left\{s_{i}\right\}$, and again all paths from the basic step in $\mathcal{A}_{1}$ pass through all levels in $G_{i, j}$. Therefore, $\mathcal{B}_{i, j}$ is a bramble for $G_{i, j}$. 
Let $C \subset V$ be a vertex set that determined the vertex connectivity of the pair $s_{i}, t_{j}$. Clearly, $C \cup\left\{s_{i}\right\}$ forms a cover for the constructed bramble. A set of representative vertices, one from each level $k, i \leq k \leq R-j$, together with $s_{i}$ also form a cover for the constructed bramble. Thus, the order of the bramble is at $\operatorname{most} \min \left\{c_{i, j}+1\right.$, $j-i+2\}$. Since the levels are non-intersecting, the paths constructed at the basic step of $\mathcal{A}_{1}$ are vertex disjoint, and the bramble contains all combinations of these levels and paths, the order of the bramble is at least $\min \left\{c_{i, j}+1, j-i+2\right\}$, which completes the proof.

Note that, although $G_{i, j}$ is a minor of $G$ if $j<R-1$, the bramble cannot be extended with subset $\left\{t_{j}\right\}$ since $s_{i}$ and $t_{j}$ are not touching.

Corollary 2.2 For each graph $G, L B_{1} \leq t w(G)$.

Proof By Theorem 2.1, each time bestlow is increased, it is set to the order of a bramble of a graph $G_{i, j}$ minus one. By Corollary 1.5 , this value is a lower bound on the treewidth of $G_{i, j}$, and as $G_{i, j}$ is a minor of $G$, this is also a lower bound on the treewidth of $G$.

Theorem 2.3 Algorithm $\mathcal{A}_{1}$ can be carried out in $O\left(n^{3} m\right)$ time on a graph $G$ with $n$ vertices and $m$ edges.

Proof Computing the partition of $V$ into the level sets requires at most $R$ constructions of a connectivity closure. The proposed procedure for this takes at most $n$ computations of shortest paths from a source to all other nodes in the graph. Each computation of a shortest path can be done in $O(n+m)$ time. We can use a straightforward implementation of Dijkstra's algorithm (see [1, pp. 108-112]), and note that, because all edge lengths are 0 or 1 , the values in the priority queue that are not $\infty$ differ by at most one. Thus, each of the $O(n+m)$ extract-min or update operations to the priority queue can be done in $O(1)$ time, and the total time of this shortest path computation costs $O(n+m)$ time. Hence, the initialization requires at most $O(R n m)$ time.

In the basic step of the algorithm we compute $c$ vertex disjoint $(s, t)$-paths in a graph, that can be done in $O(\mathrm{~nm})$ time; see [1, pp. 273-277]. Together with enumeration over all possibilities for $i$ and $j$, it brings the time complexity of the basic step up to $O\left(R^{2} n m\right)$. Since $R \leq n$, we have that the total running time of $\mathcal{A}_{1}$ is at most $O\left(n^{3} m\right)$, as required.

At cost of an additional multiplicative factor of $n$ we can find a root vertex $r$ for the BFS that provides the best lower bound for the treewidth, cf. Sect. 4 .

\section{Brambles in Planar Graphs}

Algorithm $\mathcal{A}_{1}$ can be significantly improved in running time when the input graph is restricted to be planar. Given an embedding of $G_{i, j} \backslash\left\{s_{i}, t_{j}\right\}$, the vertex disjoint paths $P_{\ell}$ can be viewed as layers in the graph, with the remaining vertices laying 
between (below/above) these layers. Since every level set $V_{i}$ is connected as well and all paths intersect with $V_{i}$, the vertices in between the layers can be contracted either to the layer above or below. If we further contract vertices on a path that belong to the same level set $V_{i}$, the resulting graph contains a $c_{i j}$ by $j-i+1$ grid, and hence the treewidth of $G_{i, j}$ is at least $\min \left\{c_{i j}, j-i+1\right\}$. The need for a root vertex $r$, however, causes that for grid graphs the treewidth may not be reached by Algorithm $\mathcal{A}_{1}$.

In this section we present a different algorithm that searches for grid minors in planar graphs more directly. Algorithm $\mathcal{A}_{2}$ below finds brambles in several minors of a connected planar graph $G$. In the initialization step, we partition the vertices on the exterior face in North, East, West, and South. In the basic step, the algorithm builds grid minors of $G$ for which we know how the maximum order bramble looks like. Algorithm $\mathcal{A}_{2}$ outputs the largest lower bound due to the grid minors and corresponding brambles it has met.

\section{Algorithm $\mathcal{A}_{2}$}

Input. A planar embedding of $G$ with no edge crossings. It is well known that such an embedding can be constructed in linear time (e.g., the $O(n+m)$ algorithm by Hopcroft and Tarjan [26]).

Initialization. Let $F$ be the exterior face of the embedding and let $f$ be the length of a single closed clockwise walk along $F$. Since $G$ is a simple graph, $f \geq|F| \geq 3$. Let North, East, South, and West be four paths on $F$ (possibly atomic if $F$ is a simple cycle on 3 vertices) being sequential parts of a closed clockwise walk along $F$ such that North has one common endpoint with East, East has one common endpoint with South, South with West, and finally West with North. Moreover, let the lengths of these four paths be roughly the same, i.e., the length may vary by at most one vertex being either $\lfloor f / 4\rfloor+1$ or $\lceil f / 4\rceil+1$. Notice that such paths always exist and can be found in linear time. We add in the exterior four dummy vertices $\mathcal{N}, \mathcal{E}, \mathcal{S}$, and $\mathcal{W}$ and connect them to all vertices in North, East, South, and West respectively. Further in the paper we always refer to the vertices incident to $\mathcal{N}, \mathcal{E}, \mathcal{S}, \mathcal{W}$ as to North, East, South and West, respectively; and we denote Dummy $=\{\mathcal{N}, \mathcal{E}, \mathcal{S}, \mathcal{W}\}$. Finally, we set integer variable bestlow to -1 .

Basic step. We view the algorithm as a rooted search tree with a root corresponding to the graph constructed in the initialization. At each node of the search tree we perform the following steps.

1. Given a node $i$ in the tree and the planar graph $G_{i}=\left(V_{i}, E_{i}\right)$ associated with this node. Let $c_{i}$ be the vertex connectivity of $(\mathcal{N}, \mathcal{S})$ in graph $G_{i} \backslash\{\mathcal{E}, \mathcal{W}\}$ and $d_{i}$ the vertex connectivity of $(\mathcal{W}, \mathcal{E})$ in $G_{i} \backslash\{\mathcal{N}, \mathcal{S}\}$. Find $c_{i}$ vertex disjoint paths connecting $\mathcal{N}$ and $\mathcal{S}$, and $d_{i}$ vertex disjoint paths connecting $\mathcal{W}$ and $\mathcal{E}$. Clearly, $G_{i} \backslash$ Dummy contains a $c_{i} \times d_{i}$ grid as a minor, and therefore it contains also a $b_{i} \times b_{i}$ grid as a minor, where $b_{i}=\min \left\{c_{i}, d_{i}\right\}$. Create an order $b_{i}+1$ bramble $\mathcal{B}_{i}$ for this $b_{i} \times b_{i}$ grid as described in Section 1. Set bestlow to the maximum of bestlow and $b_{i}$.

2. If $b_{i}=c_{i}$, we create the child nodes of $i$ as follows. Let $C_{i} \subset V_{i}$ be a vertex set determining the vertex connectivity of $(\mathcal{N}, \mathcal{S})$ in $G_{i} \backslash\{\mathcal{E}, \mathcal{W}\}$. Further in the text we refer to such a set as a separator. The graph $G_{i} \backslash\left(C_{i} \cup\{\mathcal{E}, \mathcal{W}\}\right)$ has at least 
two components, one containing $\mathcal{N}$ and another containing $\mathcal{S}$. Notice that there can be other components eventually separated by $C_{i}$ from both $\mathcal{N}$ and $\mathcal{S}$. For each component we create a child node in the search tree and construct the graph associated with this child node as follows.

Let $G_{i}^{q}$ be a component in $G_{i} \backslash\left(C_{i} \cup\{\mathcal{E}, \mathcal{W}\}\right)$. Consider the exterior face $X$ of $G_{i}^{q} \backslash$ Dummy. Now, like in the initialization, we define four paths North, East, South, and West in a single closed clockwise walk along $X$. If a vertex $v \in X$ belongs to North in $G_{i}$, let this vertex belong to North in $G_{i}^{q}$ as well. In the similar way we leave the vertices which are already assigned to East, South, and West in $G_{i}$ in the corresponding paths in $G_{i}^{q}$.

Yet unassigned vertices in $X$ we assign to the paths in such a way that, again, the paths (possibly atomic) become sequential parts of a closed clockwise walk along $X$ such that North has one common endpoint with East, East has one common endpoint with South, South with West, and West with North. Finally, we connect $\mathcal{N}$ to North, $\mathcal{E}$ to East, $\mathcal{S}$ to South, and $\mathcal{W}$ to West. Let the resulting graph be a graph associated with a child node of $i$ corresponding to component $G_{i}^{q}$.

3 . If $d_{i}<c_{i}$, we similarly create the child nodes with respect to the vertex connectivity of $(\mathcal{E}, \mathcal{W})$.

4. We recurse on the child nodes unless the number of non-dummy vertices in the graph associated with the node becomes less or equal to the current value of bestlow.

Output. Output $L B_{2}=$ bestlow. STOP.

Theorem 3.1 The lower bound $\mathrm{LB}_{2}$ on the treewidth of a planar graph can be obtained by Algorithm $\mathcal{A}_{2}$ in time $O\left(n^{2} \log n\right)$.

Proof We already observed that for any node $i$ in the search tree, $b_{i}$ is the size of a side of a square grid minor in $G$. Since the treewidth of a square grid is equal to the size of a side of the grid, we directly have that bestlow $=\max _{i} b_{i}$ is a lower bound on the treewidth of $G$.

Notice that the initialization requires only linear time. In the basic step of the algorithm we compute two vertex connectivities in a planar network, which can be done in $O(n \log n)$ time; see Theorem 8.8 in [1, p. 265]. Then we create the child nodes in the search tree basically determining the connected components in a graph which takes linear time. Therefore, the basic step of the algorithm requires $O(n \log n)$ time.

We perform the basic step for all nodes of the search tree. Let us argue that the number of nodes in this tree is at most $n$. Consider leaf nodes of the tree. By construction, the vertex sets of the graphs associated with all leaf nodes are mutually disjoint. Now, consider the immediate parent node of some nodes. The graph associated with this parent node contains a vertex set of $G$ (determining the vertex connectivity of two dummy vertices at a certain basic step of the algorithm) which is removed from all child nodes. Therefore, moving up to the root, in each parent node we find at least one vertex which is not present in any of its children. Since the number of vertices in $V$ is $n$, we conclude, that the search tree has size at most $n$. 

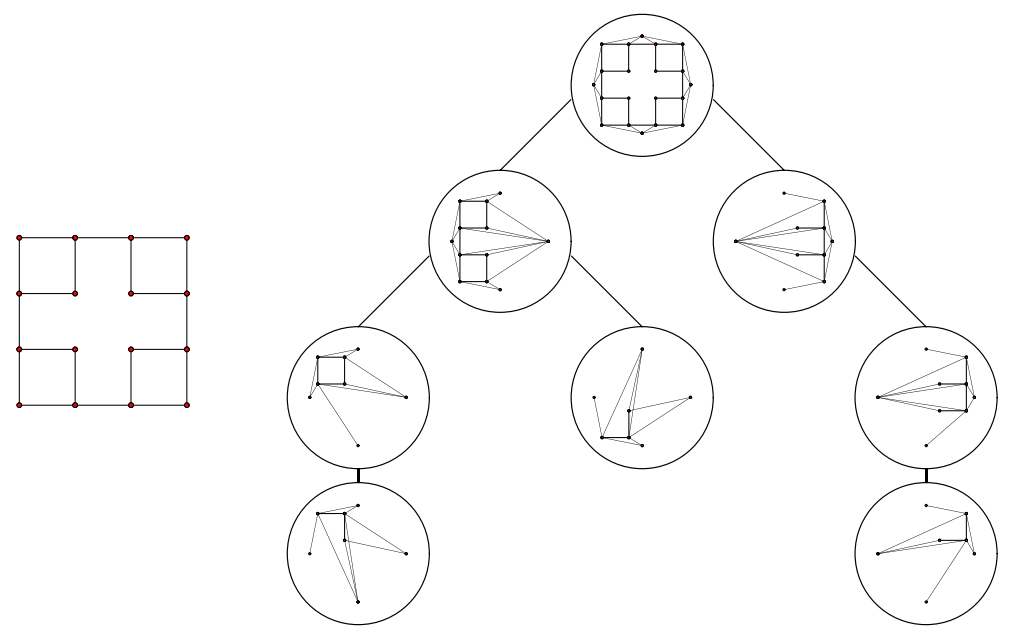

(a) graph

(b) rooted tree

Fig. 2 Graph and rooted tree of Algorithm $\mathcal{A}_{2}$ (trivial child nodes are left out)

Thus, applying the basic step to all nodes of the tree, we have the total running time of $\mathcal{A}_{2}$ at most $O\left(n^{2} \log n\right)$, as required.

It is noticeable that Algorithm $\mathcal{A}_{2}$, besides estimation of the treewidth, approximates another parameter of the planar graph, namely the size of a side in the largest grid minor. It is well known that planar graphs having a large treewidth must have also a large grid as a minor; see e.g. $[17,19,36]$. The following theorem and corollary present the algorithmic consequences of this fact.

Theorem 3.2 For any planar graph $G$, the lower bound on the treewidth returned by Algorithm $\mathcal{A}_{2}$ satisfies the inequality $b / 4 \leq L B_{2} \leq b$, where $b$ is the size of a side in the largest square grid minor in $G$.

Proof Let $M$ be the largest square grid minor in $G$ with the size of a side $b$. By construction, $L B_{2}$ is the size of a side in a square grid minor of $G$. Therefore, inequality $L B_{2} \leq b$ always holds. Thus, it remains to prove the lower bound for $L B_{2}$.

Consider a node $i$ in the search tree such that $G_{i}$ contains at least $b^{2} / 2$ vertices of $M$ and each of its children contains at most $b^{2} / 2$ vertices of $M$. Notice that such a node does always exist otherwise we have a contradiction to the stoppage criteria of the algorithm.

In each node of the search tree, the graphs associated with its child nodes are some connected components in $G \backslash S$ where $S$ consists of at most four vertex cuts obtained in the parent nodes (at most two vertex cuts determining $(\mathcal{N}, \mathcal{S}$ ) connectivity and at most two vertex cuts determining $(\mathcal{E}, \mathcal{W})$ connectivity). Let $S_{i}$ be the union of these vertex cuts determining the child nodes of $i$, i.e. the child nodes of $i$ are some connected components in $G \backslash S_{i}$. By definition of node $i$, we have that $G \backslash\left(V_{i} \cup S_{i}\right)$ 
contains at most $b^{2} / 2$ vertices of $M$ and each of the child nodes of $i$ contains also at most $b^{2} / 2$ vertices of $M$.

For a graph $G^{\prime}=\left(V^{\prime}, E^{\prime}\right)$, let us refer to a subset $S \subseteq V^{\prime}$ as to balanced separator of $G^{\prime}$ if each connected component in $G^{\prime} \backslash S$ has at most $\left|V^{\prime}\right| / 2$ vertices. It is well known that a minimum balanced separator in a $b \times b$ grid graph has size $b$, see e.g. [18] (the size of a minimum balanced separator is also known as the vertex bisection of the graph). From observation above we have that $S_{i} \cap M$ is a balanced separator in $M$, and therefore $\left|S_{i}\right| \geq b$. Since $S_{i}$ consists of at most 4 vertex cuts, one of these cuts must have size at least $b / 4$ that completes the proof.

From Theorem 3.2 and the result by Robertson, Seymour, and Thomas [36] that every planar graph of treewidth $t w(G)$ has an $\Omega(t w(G)) \times \Omega(t w(G))$ grid graph as a minor, we deduce the following corollary.

Corollary 3.3 Algorithm $\mathcal{A}_{2}$ is a constant approximation algorithm for the treewidth and for the branchwidth of planar graphs.

Proof From Theorem 3.2 we have that $S=\Theta\left(L B_{2}\right)$. From the result by Robertson, Seymour, and Thomas [36], we have that $t w(G)=\Theta(b w(G))=\Theta(S)=\Theta\left(L B_{2}\right)$, where $b w(G)$ is the branchwidth of graph $G$.

We complete this section with a brief discussion of advantages and disadvantages of Algorithm $\mathcal{A}_{2}$ in comparison with the known approximation algorithms for the treewidth on planar graphs. Seymour and Thomas have shown that the branchwidth of a planar graph can be computed in polynomial time [38]. By the result of Robertson and Seymour [34] that each branch decomposition can be converted to a tree decomposition with width at most $3 / 2$ times the width of the branch decomposition (cf. Sect. 1), we directly have a polynomial time approximation algorithm for treewidth of planar graphs with ratio $3 / 2$.

The decision version of the algorithm of Seymour and Thomas [38] runs in $O\left(n^{2}\right)$ time. A version that also constructs branch decompositions of optimal width uses more time; recently, Gu and Tamaki [23] showed this can be done in $O\left(n^{3}\right)$ time. If we want to obtain lower bounds on the treewidth, we only need to run the decision version. However, an important disadvantage of the Seymour and Thomas algorithm is that it is not memory friendly and even for the medium size graphs it easily runs out of memory; see [24, 25]. Recently, Hicks in [25] made several attempts to get a memory friendly algorithm based on the Seymour and Thomas ideas. He derived two memory friendly algorithms with running time $O\left(n^{3}\right)$. Clearly, the machinery of Algorithm $\mathcal{A}_{2}$ does not require much of memory resources and the algorithm has nearly the same running time as Seymour and Thomas' algorithm, and better running time than Hicks' algorithm. The disadvantage of Algorithm $\mathcal{A}_{2}$ is its worse performance ratio in comparison to the other algorithms. On the other hand, Algorithm $\mathcal{A}_{2}$ eventually estimates another important parameter of a planar graph, namely the size of a side in the largest square grid minor. Moreover, the version of Algorithm $\mathcal{A}_{2}$ that also constructs a square grid minor with the size of a side at least $1 / 4$ of the side size in the largest grid minor, runs in the same $O\left(n^{2} \log n\right)$ time. 


\section{Computational Experiments}

In order to compare in practice the quality of Algorithms $\mathcal{A}_{1}$ and $\mathcal{A}_{2}$ with previously studied treewidth lower bounds, these algorithms have been implemented in $\mathrm{C}++$ and tried out on a collection of input graphs. In this section, we report on the obtained results for two selected sets of instances as well as for grid graphs. The first set contains a number of general graphs, that have been used in previous studies $[10,28]$ and originate from different applications like probabilistic networks, frequency assignment, and vertex coloring. The second set of instances consists of planar graphs that have been used by Hicks [24, 25] before. From both sets we selected some instances that are representative for the whole set and/or show an interesting behavior. The CPU times reported are in seconds and obtained on a Linux-operated PC with $3.0 \mathrm{GHz}$ Intel Pentium 4 processor. Some extensive experimental results can be found on [39].

Algorithm $\mathcal{A}_{1} \quad$ Algorithm $\mathcal{A}_{1}$ for general graphs has been tested on both the selected planar and non-planar graphs. In the implementation, we skip a graph $G_{i, j}$, whenever $j-i+1$ is not larger than the current value of bestlow, as such graphs cannot help to increase the lower bound. This speeds up the algorithm considerably.

For grid graphs, Algorithm $\mathcal{A}_{1}$ can be analyzed. The best result can be achieved by taking all vertices as root vertex $r$ once. Now consider an $x \times x$ grid (i.e., treewidth equals $x$ ). For this instance, the maximum is achieved when taking $r$ the mid point of a side of the grid (without loss of generality we assume that $x$ is an odd number). Here, the BFS level $V_{i}, 1 \leq i \leq x / 2$, is a path of length $4 i+1$ and the BFS level $V_{i}$, $x / 2<i \leq x-1$ is simply a column/row of the grid (i.e., for a vertex $r$ at the border of the grid, the sets $V_{i}$ are defined by all vertices on distance $i$ measured in the $L_{\infty^{-}}$ norm). In graph $G_{i, x-1}, 1 \leq i \leq\lfloor x / 2\rfloor$, there are $\min \{4 i-1, x\}$ vertex disjoint paths between $s_{i}$ and $t_{x-1}$. In graph $G_{i, x-1},\lceil x / 2\rceil \leq i \leq x-1$, there are exactly $x$ vertex disjoint paths between $s_{i}$ and $t_{x-1}$. Therefore, the value bestlow will be updated with $\min \{4 i-1, x-i\}$. Thus, maximizing the minimum over $i$, we derive that at the end of the algorithm the value bestlow is at least $\lfloor 4(x-1) / 5\rfloor$, i.e., roughly $4 / 5$ of the treewidth. This explains the value of 19 for $25 \times 25$ grid reported in Table 2 .

The additional $O(n)$ complexity of the algorithm can be reduced in practice by sorting or limiting the number of root vertices. In principle the algorithm has to be executed for only one of the vertices for which the maximum is achieved. However, we cannot select on this value before computing it. Experiments have shown that the eccentricity of a vertex is a reasonable criterion to sort/limit the root vertices. The eccentricity $\varepsilon(v)$ of a vertex $v$ is the maximum depth of a breadth first search with $v$ as root, cf. [40] and hence the best lower bound with root $r$ is limited by $\varepsilon(r)$. Figure 3 shows exemplarily for two planar graphs the eccentricity and $L B_{1}$ for all possible root vertices, sorted by decreasing eccentricity with $L B_{1}$ as tiebreaker (highest first). If the best bound achieved so far is at least $\varepsilon(r)$ for some $r \in V$, we do not have to run algorithm $\mathcal{A}_{1}$ with $r$ as root. By sorting the vertices according to decreasing eccentricity, the number of root vertices for which the algorithm should be executed to obtain the best $L B_{1}$ is limited in our computations this way. Figure 3 in fact indicates that the computation times can be reduced further by limiting the number of root vertices to those with high eccentricity, since it is very unlikely that vertices with $\varepsilon(v)$ not close to the maximum achieve the maximum $L B_{1}$. 


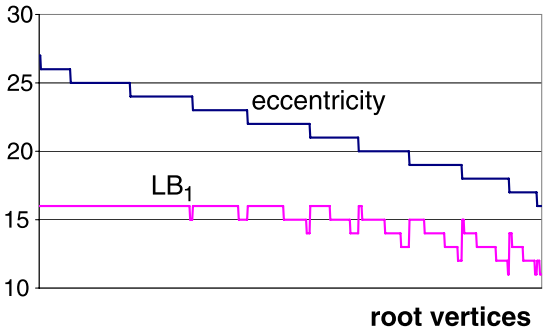

(a) $u 724$

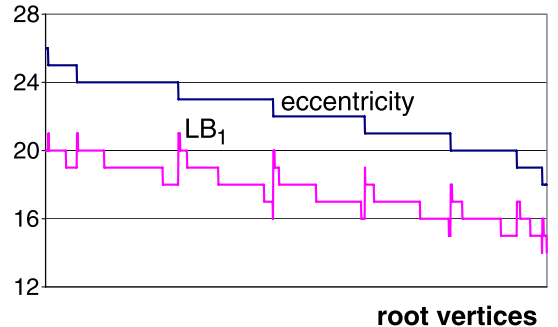

(b) nrw1379

Fig. $3 L B_{1}$ and eccentricity for all possible root vertices, sorted according to decreasing eccentricity with $L B_{1}$ as tiebreaker (highest first)

Table 1 Results for Algorithm $\mathcal{A}_{1}$ on selected non-planar graphs. The last five instances are subproblems within the tour merging algorithm for TSP [16] and are close to planar. They have been pre-processed by the rules in [11] to reduce the size of the graphs

\begin{tabular}{lrrrrrrr}
\hline Instance & $|V|$ & $|E|$ & $\delta C$ & CPU & $L B_{1}$ & CPU & $U B$ \\
\hline link & 724 & 1738 & 11 & 0.02 & 7 & 89.95 & 13 \\
munin1 & 189 & 366 & 10 & 0.00 & 4 & 2.19 & 11 \\
munin3 & 1044 & 1745 & 7 & 0.01 & 4 & 195.35 & 7 \\
pignet2 & 3032 & 7264 & 38 & 0.12 & 5 & 3456.77 & 135 \\
celar06 & 100 & 350 & 11 & 0.00 & 3 & 1.50 & 11 \\
celar07pp & 162 & 764 & 15 & 0.01 & 3 & 19.40 & 18 \\
graph04 & 200 & 734 & 20 & 0.02 & 5 & 0.38 & 55 \\
school1 & 385 & 19095 & 122 & 0.59 & 3 & 46.22 & 188 \\
school1-nsh & 352 & 14612 & 106 & 0.39 & 3 & 45.34 & 162 \\
zeroin.i.1 & 126 & 4100 & 50 & 0.04 & 2 & 0.30 & 50 \\
& & & & & & & \\
fl3795-pp & 1433 & 3098 & 6 & 0.04 & 6 & 1501.53 & 13 \\
fn14461-pp & 1528 & 3114 & 5 & 0.05 & 14 & 4703.67 & 33 \\
pcb3038-pp & 948 & 1920 & 5 & 0.03 & 12 & 383.77 & 25 \\
rl5915-pp & 863 & 1730 & 5 & 0.02 & 10 & 470.76 & 23 \\
rl5934-pp & 904 & 1800 & 5 & 0.02 & 12 & 378.17 & 23 \\
\hline
\end{tabular}

Table 1 reports the results for non-planar graphs. For comparison a contraction degeneracy $\delta C(G)$ lower bound [10] is reported as well. Other recently studied bounds, like MCSLB $[8,29]$ are outperformed by this bound or require significantly more time for a slightly better bound. See also [39].

Different behavior can be observed: for the graphs originating from probabilistic networks, frequency assignment, and coloring, $L B_{1}$ is outperformed by the contraction degeneracy, both in time and value. For the graphs originating from a solution approach for the traveling salesman problem, $L B_{1}$ is significantly higher than $\delta C(G)$. It is known that these graphs are close to planar, which restricts the contraction degeneracy to exceed small values (i.e., $\delta C(G) \leq 5+\gamma(G)$, where $\gamma(G)$ is the genus of 


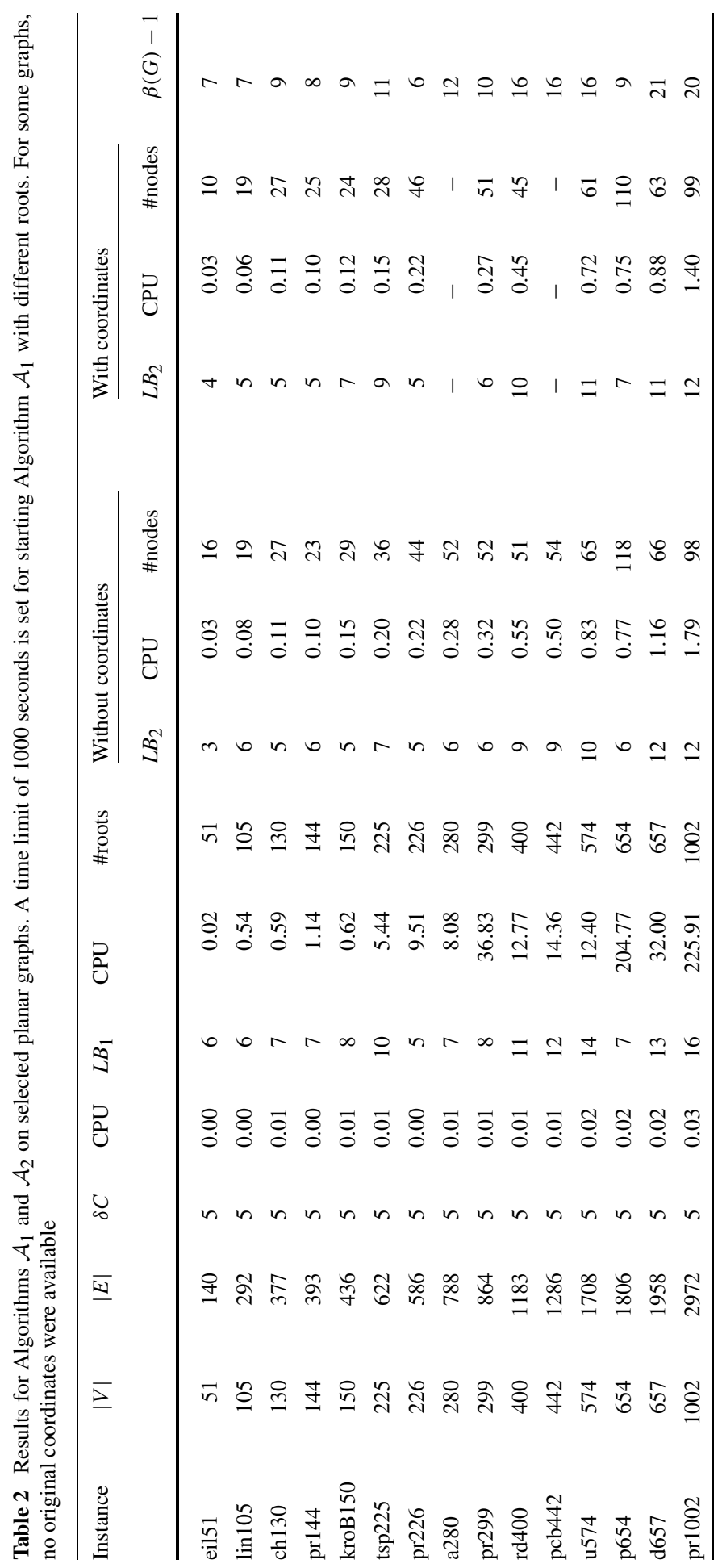




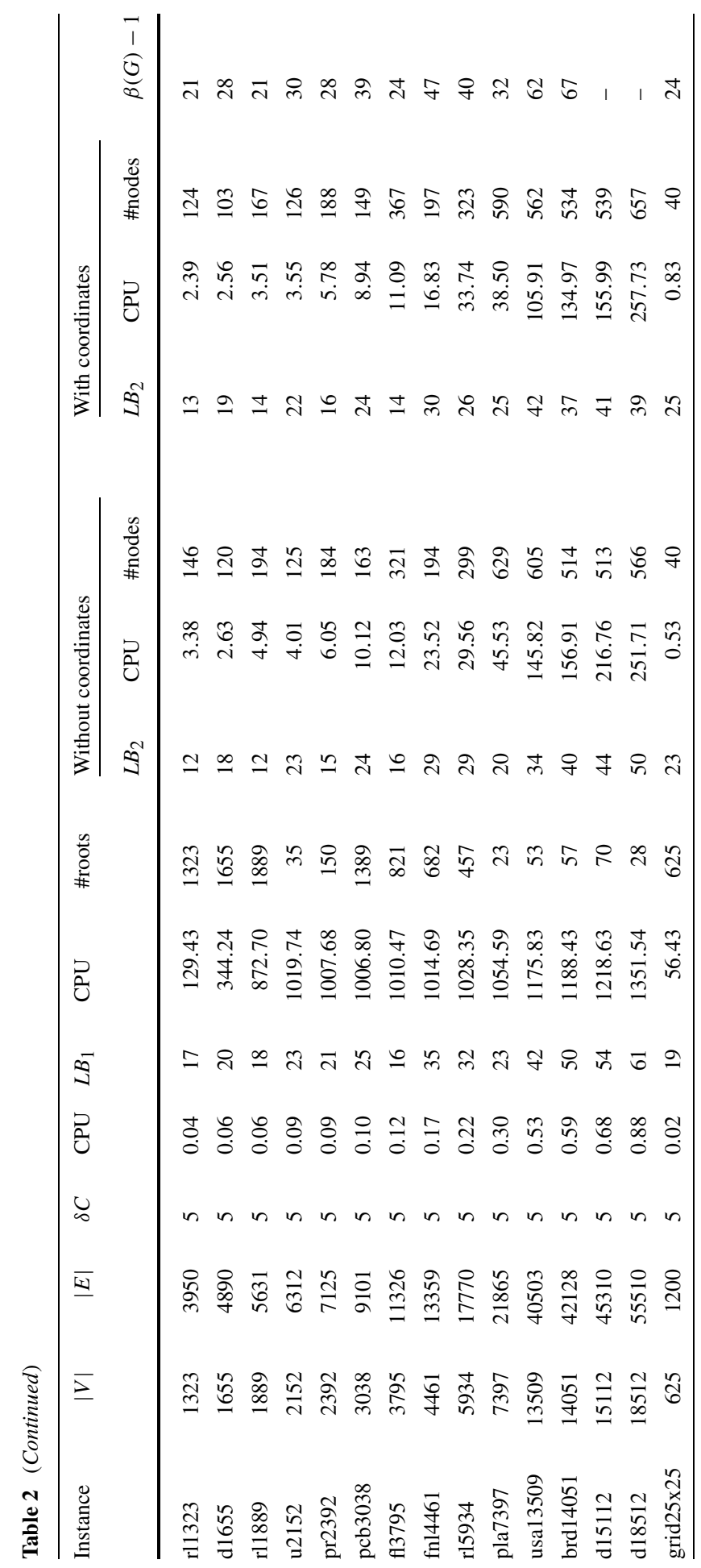


$G$ [41]). As was hoped for, the new lower bound turns out to be profitable for exactly those instances, closing the gap to the best known upper bound $U B$ substantially.

Table 2 shows the results of Algorithm $\mathcal{A}_{1}$ for planar graphs. The number of restarts of the algorithm with other roots is restricted by an overall time limit of 1000 seconds. This limit does not include the computation of the eccentricity; the reported CPU times includes this limit. The column "\#roots" displays the number of restarts within the time limit. Experiments have shown that further restarts have a very limited effect on the returned lower bound. For these graphs, the same behavior as for close to planar graphs can be observed: the lower bound is substantially increased.

Algorithm $\mathcal{A}_{2}$ For grid graphs, the result of Algorithm $\mathcal{A}_{2}$ depends on the way the exterior face is partitioned. If the partition matches exactly the sides of the grid, the algorithm returns the size of the smallest side which is in fact the treewidth. If the partition is unlucky in the sense that each side of the grid is partitioned among two of the extra vertices, the returned value can drop to half the treewidth. This can be avoided easily by rotating the partition and rerunning the algorithm.

Table 2 also shows the lower bound $L B_{2}$ computed by algorithm $\mathcal{A}_{2}$. Results are presented with and without usage of the original coordinates. If no coordinates are used, the longest face of the computed planar embedding is taken as initial outer face. With coordinates, the outer face is computed by a "walk around" starting at the vertex with lexicographic highest coordinates. The face is partitioned in (roughly) equally sized parts North, East, South, and West. Rotation has not been applied for these instances. The results with or without coordinates are inconsistent. In some cases it is advantageous to have coordinates, in other cases it is not.

Comparison Comparing $L B_{1}$ and $L B_{2}$, there is no clear winner. In some cases $L B_{2}$ is better than $L B_{1}$, but more often it is slightly worse. Since the computation time of $L B_{1}$ is the sum of a number of restarts as well as the eccentricity computation, it is difficult to compare the algorithms in this respect.

The 3/2-approximation algorithm of Seymour and Thomas [38] for planar graphs computes in fact the branchwidth $\beta(G)$. It is well-known that the $\beta(G)-1$ is a lower bound on the treewidth. The values reported in Table 2 are taken from Hicks [24]. In all cases, this lower bound is higher than $L B_{1}$ and $L B_{2}$, as is the computational effort, cf. [24]. For the two largest graphs, Hicks did not report the branchwidth; for other large graphs the computational effort is significantly higher than that of our algorithms. Where memory consumption is in issue in the Ratcatcher algorithms of Hicks, our algorithms have moderate space requirements.

\section{Concluding Remarks}

The treewidth of a graph can be characterized by the notion of brambles, introduced by Seymour and Thomas [38]. In this work, we developed bramble construction algorithms as to bound the treewidth from below. The constructed brambles turn out to be profitable, both in theory and practice, for graphs that are (close to) planar. These results complement previously studied treewidth lower bounds that turned out to be good for graphs that are far from planar. 
The brambles searched for in this paper have structures that allow easy construction and order computation. An interesting question for further research is whether more such structures exist. These may perhaps lead to new lower bound algorithms for treewidth. Experiments (see e.g., [39]) reveal that for many instances, taken from applications, there still are significant gaps between the best upper and lower bounds, especially for graphs that are (close to) planar; thus, while this paper makes a useful step, the quest for better algorithms for determining or approximating the treewidth of such graphs remains.

Acknowledgements We thank Illya Hicks for providing us with the planar graphs for our experiments, and an anonymous referee for pointing out a faster running time for the initialization step of Algorithm $\mathcal{A}_{1}$.

\section{References}

1. Ahuja, R.K., Magnanti, T.L., Orlin, J.B.: Network Flows: Theory, Algorithms, and Applications. Prentice Hall, New York (1993)

2. Amir, E.: Efficient approximations for triangulation of minimum treewidth. In: Proceedings of the 17th Conference on Uncertainty in Artificial Intelligence, pp. 7-15, 2001

3. Arnborg, S., Corneil, D.G., Proskurowski, A.: Complexity of finding embeddings in a $k$-tree. SIAM J. Algebr. Discrete Methods 8, 277-284 (1987)

4. Bellenbaum, P., Diestel, R.: Two short proofs concerning tree-decompositions. Comb. Probab. Comput. 11, 541-547 (2002)

5. Bodlaender, H.L.: A linear time algorithm for finding tree-decompositions of small treewidth. SIAM J. Comput. 25, 1305-1317 (1996)

6. Bodlaender, H.L.: A partial $k$-arboretum of graphs with bounded treewidth. Theor. Comput. Sci. 209, $1-45(1998)$

7. Bodlaender, H.L.: Discovering treewidth. In: Vojtás̆, P., Bieliková, M., Charron-Bost, B. (eds.) SOFSEM 2005: Theory and Practive of Computer Science: 31st Conference on Current Trends in Theory and Practive of Computer Science. Lecture Notes in Computer Science, vol. 3381, pp. 1-16. Springer, Berlin (2005)

8. Bodlaender, H.L., Koster, A.M.C.A.: On the Maximum Cardinality Search lower bound for treewidth. In: Hromkovič, J., Nagl, M., Westfechtel, B. (eds.) Proc. 30th International Workshop on GraphTheoretic Concepts in Computer Science WG 2004. Lecture Notes in Computer Science, vol. 3353, pp. 81-92. Springer, Berlin (2004)

9. Bodlaender, H.L., Koster, A.M.C.A.: Safe separators for treewidth. Discrete Math. 306, 337-350 (2006)

10. Bodlaender, H.L., Koster, A.M.C.A., Wolle, T.: Contraction and treewidth lower bounds. In: Albers, S., Radzik, T. (eds.) Proceedings 12th Annual European Symposium on Algorithms, ESA2004. Lecture Notes in Computer Science, vol. 3221, pp. 628-639. Springer, Berlin (2004)

11. Bodlaender, H.L., Koster, A.M.C.A., Eijkhof, F.v.d: Pre-processing rules for triangulation of probabilistic networks. Comput. Intell. 21(3), 286-305 (2005)

12. Bodlaender, H.L., Koster, A.M.C.A., Wolle, T.: Contraction and treewidth lower bounds. J. Graph Algorithms Appl. 10(1), 5-49 (2006)

13. Bouchitté, V., Todinca, I.: Treewidth and minimum fill-in: Grouping the minimal separators. SIAM J. Comput. 31, 212-232 (2001)

14. Clautiaux, F., Carlier, J., Moukrim, A., Négre, S.: New lower and upper bounds for graph treewidth. In: Rolim, J.D.P. (ed.) Proceedings International Workshop on Experimental and Efficient Algorithms, WEA 2003. Lecture Notes in Computer Science, vol. 2647, pp. 70-80. Springer, Berlin (2003)

15. Clautiaux, F., Moukrim, A., Négre, S., Carlier, J.: Heuristic and meta-heuristic methods for computing graph treewidth. RAIRO Oper. Res. 38, 13-26 (2004)

16. Cook, W., Seymour, P.D.: Tour merging via branch-decomposition. INFORMS J. Comput. 15(3), 233-248 (2003)

17. Demaine, E.D., Hajiaghayi, M.: Graphs excluding a fixed minor have grids as large as treewidth, with combinatorial and algorithmic applications through bidimensionality. In: Proceedings of the 16th Annual ACM-SIAM Symposium on Discrete Algorithms, SODA2005, pp. 682-689, 2005 
18. Díaz, J., Petit, J., Serna, M.: A survey of graph layout problems. ACM Comput. Surv. 34, 313-356 (2002)

19. Diestel, R., Jensen, T.R., Gorbunov, K.Y., Thomassen, C.: Highly connected sets and the excluded grid theorem. J. Comb. Theory Ser. B 75, 61-73 (1999)

20. Fomin, F.V., Kratsch, D., Todinca, I.: Exact (exponential) algorithms for treewidth and minimum fillin. In: Proceedings of the 31st International Colloquium on Automata, Languages and Programming, pp. 568-580, 2004

21. Garey, M.R., Johnson, D.S.: Computers and Intractability, A Guide to the Theory of NPCompleteness. Freeman, New York (1979)

22. Gogate, V., Dechter, R.: A complete anytime algorithm for treewidth. In: Proceedings of the 20th Annual Conference on Uncertainty in Artificial Intelligence UAI-04, pp. 201-208, Arlington, Virginia, USA. AUAI Press (2004)

23. Gu, Q.-P., Tamaki, H.: Optimal branch-decomposition of planar graphs in $O\left(n^{3}\right)$ time. In: Proceedings of the 32nd International Colloquium on Automata, Languages and Programming. Lecture Notes in Computer Science. Springer, Berlin (2005)

24. Hicks, I.V.: Planar branch decompositions I: the Ratcatcher. INFORMS J. Comput. 17, 402-412 (2005)

25. Hicks, I.V.: Planar branch decompositions II: the cycle method. INFORMS J. Comput. 17, 413-421 (2005)

26. Hopcroft, J.E., Tarjan, R.E.: Efficient planarity testing. J. ACM 21, 549-568 (1974)

27. Koster, A.M.C.A., Bodlaender, H.L., van Hoesel, S.P.M.: Treewidth: computational experiments. In: Broersma, H., Faigle, U., Hurink, J., Pickl, S. (eds.) Electronic Notes in Discrete Mathematics, vol. 8, pp. 54-57. Elsevier, Amsterdam (2001)

28. Koster, A.M.C.A., Wolle, T., Bodlaender, H.L.: Degree-based treewidth lower bounds. In: Nikoletseas, S.E. (ed.) Proceedings of the 4th International Workshop on Experimental and Efficient Algorithms WEA 2005. Lecture Notes in Computer Science, vol. 3503, pp. 101-112. Springer, Berlin (2005)

29. Lucena, B.: A new lower bound for tree-width using maximum cardinality search. SIAM J. Discrete Math. 16, 345-353 (2003)

30. Menger, K.: Zur allgemeinen Kurventheorie. Fund. Math. 10, 96-115 (1927)

31. Ramachandramurthi, S.: The structure and number of obstructions to treewidth. SIAM J. Discrete Math. 10, 146-157 (1997)

32. Reed, B.A.: Tree Width and Tangles, a New Measure of Connectivity and Some Applications. LMS Lecture Note Series, vol. 241, pp. 87-162. Cambridge University Press, Cambridge (1997)

33. Robertson, N., Seymour, P.D.: Graph minors. II. Algorithmic aspects of tree-width. J. Algorithms 7, 309-322 (1986)

34. Robertson, N., Seymour, P.D.: Graph minors. X. Obstructions to tree-decomposition. J. Comb. Theory Ser. B 52, 153-190 (1991)

35. Robertson, N., Seymour, P.D.: Graph minors. XIII. The disjoint paths problem. J. Comb. Theory Ser. B 63, 65-110 (1995)

36. Robertson, N., Seymour, P.D., Thomas, R.: Quickly excluding a planar graph. J. Comb. Theory Ser. B 62, 323-348 (1994)

37. Seymour, P.D., Thomas, R.: Graph searching and a minimax theorem for tree-width. J. Comb. Theory Ser. B 58, 239-257 (1993)

38. Seymour, P.D., Thomas, R.: Call routing and the Ratcatcher. Combinatorica 14(2), 217-241 (1994)

39. Treewidthlib, http://www.cs.uu.nl/people/hansb/treewidthlib (2004)

40. West, D.B.: Introduction to Graph Theory. Prentice Hall, New York (2001)

41. Wolle, T., Koster, A.M.C.A., Bodlaender, H.L.: A note on contraction degeneracy. Technical Report UU-CS-2004-042, Institute of Information and Computing Sciences, Utrecht University, Utrecht, The Netherlands (2004) 